\section{OPEN ACCESS}

Edited by:

Arved Weimann,

St. Georg Hospital, Germany

Reviewed by:

Maria Wobith

St. Georg Hospital, Germany Maria Montserrat Diaz Pedrosa, State University of Maringá, Brazil

${ }^{*}$ Correspondence: Han-Ping Shi

shihp@ccmu.edu.cn

tThese authors have contributed equally to this work

Specialty section:

This article was submitted to Clinical Nutrition,

a section of the journal

Frontiers in Nutrition

Received: 24 May 2021

Accepted: 12 July 2021

Published: 04 August 2021

Citation:

Zhang X, Zhang Q, Feng L-

Zhang K-P, Tang $M$, Song $M-m$, Ruan G-t, Zhang X-w, Li W, Zhou F-X,

Cong M-H and Shi H-P (2021) The Application of Fat-Free Mass Index for Survival Prediction in Cancer Patients

With Normal and High Body Mass Index. Front. Nutr. 8:714051.

doi: 10.3389/fnut.2021.714051

\title{
The Application of Fat-Free Mass Index for Survival Prediction in Cancer Patients With Normal and High Body Mass Index
}

\begin{abstract}
Xi Zhang ${ }^{1,2,3+}$, Qi Zhang ${ }^{1,3+}$, Li-jin Feng ${ }^{4}$, Kang-Ping Zhang ${ }^{1,3}$, Meng Tang 1,3, Meng-meng Song ${ }^{1,3}$, Guo-tian Ruan ${ }^{1,3}$, Xiao-wei Zhang ${ }^{1,3}$, Wei Li ${ }^{5}$, Fu-xiang Zhou ${ }^{6}$, Ming-Hua Cong ${ }^{7}$ and Han-Ping Shi ${ }^{1,3 *}$
\end{abstract}

${ }^{1}$ Department of Gastrointestinal Surgery, Clinical Nutrition, Beijing Shijitan Hospital, Capital Medical University, Beijing, China, ${ }^{2}$ Department of Radiotherapy, Affiliated Hospital of Hebei University, Baoding, China, ${ }^{3}$ Beijing International Science and Technology Cooperation Base for Cancer Metabolism and Nutrition, Beijing, China, ${ }^{4}$ Department of Pathology, Shanghai Tenth People's Hospital, Tongji University, School of Medicine, Shanghai, China, ${ }^{5}$ Cancer Center of the First Hospital of Jilin University, Changchun, China, ${ }^{6}$ Department of Oncology, Zhongnan Hospital of Wuhan University, Wuhan University, Wuhan, China, ${ }^{7}$ Department of Comprehensive Oncology, Cancer Hospital, Chinese Academy of Medical Sciences, Beijing, China

Background: Fat-free mass (FFM) depletion can be masked by a stable body weight or weight gain in the presence of a normal or high body mass index (BMI). This study investigated the prognostic value of low fat-free mass index (FFMI) in cancer patients with normal or high BMI.

Methods: This multicenter retrospective cohort study included 1,602 cancer patients with normal/high BMI. The association of FFMI with patients' overall survival (OS) was analyzed by the Kaplan-Meier method and a Cox model.

Results: In this analysis, there were 974 (60.8\%) females and 628 (39.2\%) males. Low FFMI was associated with worse OS when compared with those patients with normal FFMI. After multivariate adjustment, low FFMI was demonstrated to be an independent unfavorable prognostic factor (HR: 1.69; 95\% Cl: 1.28, 2.23; $P<0.001$ ) in cancer patients with normal/high BMI. For specific tumor type, low FFMl was found to be associated with worse prognosis in patients with lung cancer, breast cancer and upper gastrointestinal cancer. In subgroup analysis, the association of low FFMI with worse survival was significantly modified by weight loss ( $P$ for interaction $=0.012$ ), and those patients with concurrent low FFMl and weight loss showed the worst prognosis (HR: 3.53; 95\% Cl: 2.04, 6.11; $P<0.001)$.

Conclusion: Low FFMl was associated with worse prognosis in cancer patients with normal/high BMI. This study highlights the usefulness of FFMl for prognostic estimation in these patients.

Keywords: FFMI, normal/high BMI, survival, prognosis, cancer patients 


\section{INTRODUCTION}

Cancer has been associated with fat-free mass (FFM) loss, mainly the muscle mass depletion, owing to reduced food intake, elevated energy expenditure, and excess catabolism and inflammation (1). The resulting increased muscle protein breakdown leads to a loss of muscle function and depletion of protein reserves (2). FFM loss has also been proposed to be a predictor of severe toxicity following cancer treatment (3), negative efficacy of treatment (4) and poorer survival (5). However, the simple measure of BMI or percentage of weight loss does not distinguish between the deterioration of fat and muscle mass (6). The fat-free mass index (FFMI) can be easily measured by bioelectrical impedance analysis (BIA), and it can provide far more valuable information than BMI from both functional and metabolic points of view $(7,8)$. Thus, the Global Leadership Initiative on Malnutrition (GLIM) recommended low FFMI for the muscle mass evaluation and malnutrition diagnosis (9). Previous studies have demonstrated that underweight BMI patients have a greater risk of morbidity and mortality than normal/high BMI patients $(10,11)$, and this outcome is plausible because low BMI categories include more patients with lower physiologic resilience and metabolic reserves, which are needed to withstand the catabolic burden of the tumor and the treatment process. With the growing overweight and obesity epidemic, a number of cancer patients were in the normal or even high BMI range even though they may have lost a considerable and clinically relevant amount of muscle mass (12). However, the prognostic information of low FFMI is lacking for these patients. Therefore, this large-scale retrospective cohort study was conducted to explore the effect of low FFMI on prognosis estimation in cancer patients with normal or high BMI.

\section{MATERIALS AND METHODS}

\section{Study Design and Population}

A multicenter, retrospective study was conducted in cancer patients with normal/high BMI between November 2013 and August 2018. All of the patients were admitted for cancer treatments, including surgery, chemotherapy, radiotherapy and other anti-cancer therapies. If patients experienced multiple hospitalizations, only the data for the first admission were analyzed. The inclusion criteria were: (1) age $>18$ years old; (2) length of hospital stay longer than $48 \mathrm{~h}$; (3) diagnosis of solid tumors at any stage; (4) patients presented with a BMI $\geq 18.5 \mathrm{~kg} / \mathrm{m}^{2}$ if $<70$ years old and $\geq 20.0 \mathrm{~kg} / \mathrm{m}^{2}$ if $>70$ years old. We excluded patients if they had incomplete clinical data, lacked follow-up data or reported edema or amputations. In addition, we further excluded participants with BMI $>36 \mathrm{~kg} / \mathrm{m}^{2}$, since BIA might be inaccurate in severely obese subjects (13). Ethical approval was obtained from the participating institutions through their respective institutional review boards.

\section{Patient Characteristics}

The clinicopathologic variables included age, gender, primary tumor site, pathologic stage, Karnofsky Performance Status
(KPS), alcohol consumption, smoking status, quality of life, Nutritional RiskScreening-2002 (NRS-2002) score, hemoglobin, albumin, European Organization for Research and Treatment of Cancer Quality of Life Questionnaire (EORTC QLQ-C30) summary score, neutrophil-to-lymphocyte ratio (NLR), plateletto-lymphocyte ratio (PLR) and previous treatments (surgery, chemotherapy and radiotherapy). Normal $\left(18.5-23.9 \mathrm{~kg} / \mathrm{m}^{2}\right)$, overweight $\left(24.0-27.9 \mathrm{~kg} / \mathrm{m}^{2}\right)$ and obesity $\left(\geq 28.0 \mathrm{~kg} / \mathrm{m}^{2}\right)$ BMI ranges were defined according to a reclassification of BMI for Chinese adults released by the Ministry of Health of the People's Republic of China (14). The tumor (T), node (N) and metastasis (M) categories were not included in this analysis since each tumor type has distinct T/N/M categories. The KPS data were converted to the Eastern Cooperative Oncology Group performance status (ECOG PS) using the following categories: KPS 100 (ECOG PS 0), KPS 90 to 80 (ECOG PS 1), KPS 70 to 60 (ECOG PS 2), KPS 50 to 40 (ECOG PS 3), and KPS 30 to 0 (ECOG PS 4) (15). The QLQC30 summary score is calculated as the mean of the combined 13 QLQ-C30 scale and item scores (excluding global QoL and financial impact), with a higher score indicating a better QoL $(16,17)$. All pathological staging was defined according to the 8 th edition of the AJCC TNM staging system.

\section{Body Composition and Anthropometric Measurements}

FFM was evaluated by BIA using the InBody S10 (Beijing, China) body composition analyzer. The analysis was conducted with the patients in the supine position, with two electrodes for each foot and hand attached at the four extremities. All of the procedures were conducted according to recommendations from the manufacturers (18). BMI was calculated as body weight (a weighing scale adjusted to $0.1 \mathrm{~kg}$ ) divided by the square of height. FFM can be divided by height squared to be converted into the FFM index (FFMI). Moreover, anthropometric data such as the mid-arm muscle and calf circumferences were also examined. The knee was flexed to 90 degrees with the feet and ankles relaxed, and the largest calf circumference (CC) was measured using a standard tape measure with a $0.1-\mathrm{cm}$ increment. The mid-arm circumference (MAC) and triceps skinfold (TSF) were measured in 0.1- and $1-\mathrm{mm}$ increments, respectively, at the midpoint between the acromion and the olecranon. The MAC was measured using a plastic metric tape, and the TSF was measured using skinfold calipers. The mid-arm muscle circumference (MAMC) was obtained using the following formula: $\operatorname{MAMC}(\mathrm{mm})=$ midarm circumference $(\mathrm{mm})-[3.14 \times$ triceps skinfold $(\mathrm{mm})]$. Hand grip strength (HGS) was also measured from the patient's dominant hand with a Jamar dynamometer. The patients were asked to recall what their weight was 6 months prior, and this was compared to the weight measured at the time of admission.

\section{Statistical Analysis}

All of the data are expressed as the mean \pm standard deviation, median (interquartile range, IQR), or absolute number and proportion as appropriate. Comparison of continuous variables 
TABLE 1 | Detailed baseline characteristics of the study population.

\begin{tabular}{|c|c|c|c|}
\hline \multirow[t]{2}{*}{ Characteristics } & \multirow{2}{*}{$\begin{array}{c}\text { Normal FFMI } \\
n=1,432\end{array}$} & \multirow{2}{*}{$\begin{array}{l}\text { Low FFMI } \\
\qquad n=170\end{array}$} & \multirow[t]{2}{*}{$P$-value } \\
\hline & & & \\
\hline Age, years, n (\%) & & & 0.252 \\
\hline$\leq 65$ & $1,172(81.8 \%)$ & $133(78.2 \%)$ & \\
\hline$>65$ & 260 (18.2\%) & 37 (21.8\%) & \\
\hline Gender, n (\%) & & & 0.545 \\
\hline Male & 565 (39.5\%) & $63(37.0 \%)$ & \\
\hline Female & 867 (60.5\%) & 107 (63.0\%) & \\
\hline ECOG performance status, n (\%) & & & 0.064 \\
\hline$\leq 1$ & 1,040 (72.6\%) & 112 (65.9\%) & \\
\hline$>1$ & $392(27.4 \%)$ & $58(34.1 \%)$ & \\
\hline Smoking, n (\%) & & & 0.787 \\
\hline Absent & 903 (63.1\%) & 109 (64.1\%) & \\
\hline Present & $529(36.9 \%)$ & $61(35.9 \%)$ & \\
\hline Drinking, n (\%) & & & 0.906 \\
\hline Absent & 1,174 (82.0\%) & $140(82.4 \%)$ & \\
\hline Present & $258(18.0 \%)$ & $30(17.6 \%)$ & \\
\hline TNM stages, n (\%) & & & 0.140 \\
\hline I & 299 (20.9\%) & $28(16.5 \%)$ & \\
\hline$\|$ & $370(25.8 \%)$ & $50(29.5 \%)$ & \\
\hline III & $456(31.8 \%)$ & $46(27.0 \%)$ & \\
\hline IV & 307 (21.5\%) & 46 (27.0\%) & \\
\hline M category, n (\%) & & & 0.085 \\
\hline MO & $1,135(79.3 \%)$ & 125 (73.5\%) & \\
\hline M1 & 297 (20.7\%) & 45 (26.5\%) & \\
\hline BMl category, n (\%) & & & $<0.001$ \\
\hline Normal & $676(47.2 \%)$ & $154(90.6 \%)$ & \\
\hline Overweight & $576(40.2 \%)$ & $14(8.2 \%)$ & \\
\hline Obese & $180(12.6 \%)$ & $2(1.2 \%)$ & \\
\hline NRS-score, n (\%) & & & 0.001 \\
\hline$N R S \geq 3$ & $326(22.8 \%)$ & $59(34.7 \%)$ & \\
\hline$N R S<3$ & $1,106(77.2 \%)$ & $111(65.3 \%)$ & \\
\hline Weight-loss, n (\%) & & & $<0.001$ \\
\hline Absent & $1,242(86.7 \%)$ & $127(74.7 \%)$ & \\
\hline Present & 190 (13.3\%) & $43(25.3 \%)$ & \\
\hline Reduced food intake & & & 0.025 \\
\hline Absent & $1,074(75.0 \%)$ & $114(67.1 \%)$ & \\
\hline Present & $358(25.0 \%)$ & $56(32.9 \%)$ & \\
\hline Tumor types, n (\%) & & & 0.022 \\
\hline Lung cancer & $499(34.8 \%)$ & $49(28.8 \%)$ & \\
\hline UGIC & $90(6.3 \%)$ & $21(12.4 \%)$ & \\
\hline $\mathrm{CRC}$ & $174(12.2 \%)$ & 27 (15.9\%) & \\
\hline Breast cancer & 434 (30.3\%) & 52 (30.6\%) & \\
\hline Other cancer & $235(16.4 \%)$ & $21(12.3 \%)$ & \\
\hline \multicolumn{4}{|l|}{ Previous treatments, n (\%) } \\
\hline Surgery & $883(61.7 \%)$ & $112(65.9 \%)$ & 0.284 \\
\hline Chemotherapy & $978(68.3 \%)$ & $109(64.1 \%)$ & 0.270 \\
\hline Radiotherapy & $184(12.8 \%)$ & 35 (20.6\%) & 0.005 \\
\hline \multicolumn{4}{|l|}{ QLQ-C30, median (range) } \\
\hline Summary score & 88.95 (13.84) & $87.37(18.40)$ & 0.018 \\
\hline $\mathrm{BMI}, \mathrm{kg} / \mathrm{m}^{2}$, mean $\pm \mathrm{SD}$ & $24.49 \pm 3.04$ & $21.15 \pm 2.03$ & $<0.001$ \\
\hline
\end{tabular}

(Continued)
TABLE 1 | Continued

\begin{tabular}{lccc}
\hline Characteristics & Normal FFMI & Low FFMI & \multirow{2}{*}{ P-value } \\
\cline { 2 - 3 } & $\boldsymbol{n}=\mathbf{1 , 4 3 2}$ & $\boldsymbol{n}=\mathbf{1 7 0}$ & \\
\hline MAMC, cm, mean \pm SD & $21.87 \pm 2.72$ & $20.41 \pm 2.40$ & $<0.001$ \\
MAC, cm, mean \pm SD & $27.85 \pm 3.11$ & $25.49 \pm 2.64$ & $<0.001$ \\
TSF, mm, mean \pm SD & $19.05 \pm 6.05$ & $16.18 \pm 5.24$ & $<0.001$ \\
HGS, kg, mean \pm SD & $25.19 \pm 9.19$ & $21.71 \pm 8.03$ & $<0.001$ \\
CC, cm, mean \pm SD & $35.04 \pm 3.59$ & $32.66 \pm 3.40$ & $<0.001$ \\
Albumin, g/L, mean \pm SD & $39.62 \pm 4.86$ & $39.35 \pm 5.43$ & 0.539 \\
Hemoglobin, g/L,mean \pm SD & $126.11 \pm 18.49$ & $122.53 \pm 18.35$ & 0.017 \\
NLR, median (range) & $2.27(2.09)$ & $2.48(2.74)$ & 0.017 \\
PLR, median (range) & $143.3(100.6)$ & $160.6(118.5)$ & 0.001 \\
\hline
\end{tabular}

ECOG, Eastern Cooperative Oncology Group; UGIC, upper gastrointestinal cancer; CRC, colorectal cancer; BMI, body mass index; M, metastasis; NRS-2002, Nutritional Risk Screening-2002; FFMI, fat free mass index; TSF, triceps skinfold; MAC, Mid-arm circumference; MAMC, Mid-arm muscular circumference; CC, calf-circumference; HGS, hand grip strength; NLR, neutrophil-to-lymphocyte ratio; PLR, platelet-to-lymphocyte ratio; $S D$, standard deviation.

was performed using Student's independent $t$-test, or the MannWhitney test for data without a normal distribution. Categorical variables were compared using the $\chi^{2}$-test or Fisher's exact test. The optimal cut-off values of FFMI, NLR, and PLR for survival prediction were determined with the aid of maximally selected rank statistics (19). Multivariate analysis was performed by Cox regression analysis using backward selection to identify the independent significance of different parameters. Two sensitivity analyses were performed as follows: one analysis excluded patients who died within 6 months to reduce the potential impact of reverse causation. Besides, propensity score-matching was used to control of selection bias and potential confounding (20). The propensity scores were estimated using a logistic regression model according to the following variables: age, gender, ECOG PS, pathological stage, weight loss, tumor types, radiotherapy, reduced food intake and NLR. Propensity score-matching was performed with a caliper width of 0.1 multiplied by the standard deviation for the linearly transformed propensity scores. After amending these confounding factors, we reevaluated the prognostic significance of FFMI in cancer patients with normal and high body mass index. $P<0.05$ was considered statistically significant. All of the analyses were performed using R software, version 3.6.1.

\section{RESULTS}

\section{Characteristics of Patients}

Initially, 1,812 cancer patients with normal or high BMI were enrolled in the study. Two hundred ten patients were excluded because of missing data for one or more of the variables used in our analysis. Consequently, 1,602 patients were eligible for the final analyses (Supplementary Figure 1). Table 1 summarizes the baseline characteristics of the patients. The mean age was 56.65 years old, and $39.2 \%$ were male. The patients had a mean BMI of $24.13 \mathrm{~kg} / \mathrm{m}^{2}$. The median follow-up time was 29.95 months, and 373 patients died during follow-up. 


\section{Association of the FFMI With Clinicopathologic Variables}

FFMI showed moderate positive correlations with BMI and CC, weak positive correlations with QLQ-C30 summary score,
MAMC and HGS, but showed weak negative correlations with age (Figure 1). The optimal thresholds of the FFMI were 16.3 for males and 14.5 for females (Supplementary Figure 2). Patients with low FFMI tended to present with poorer
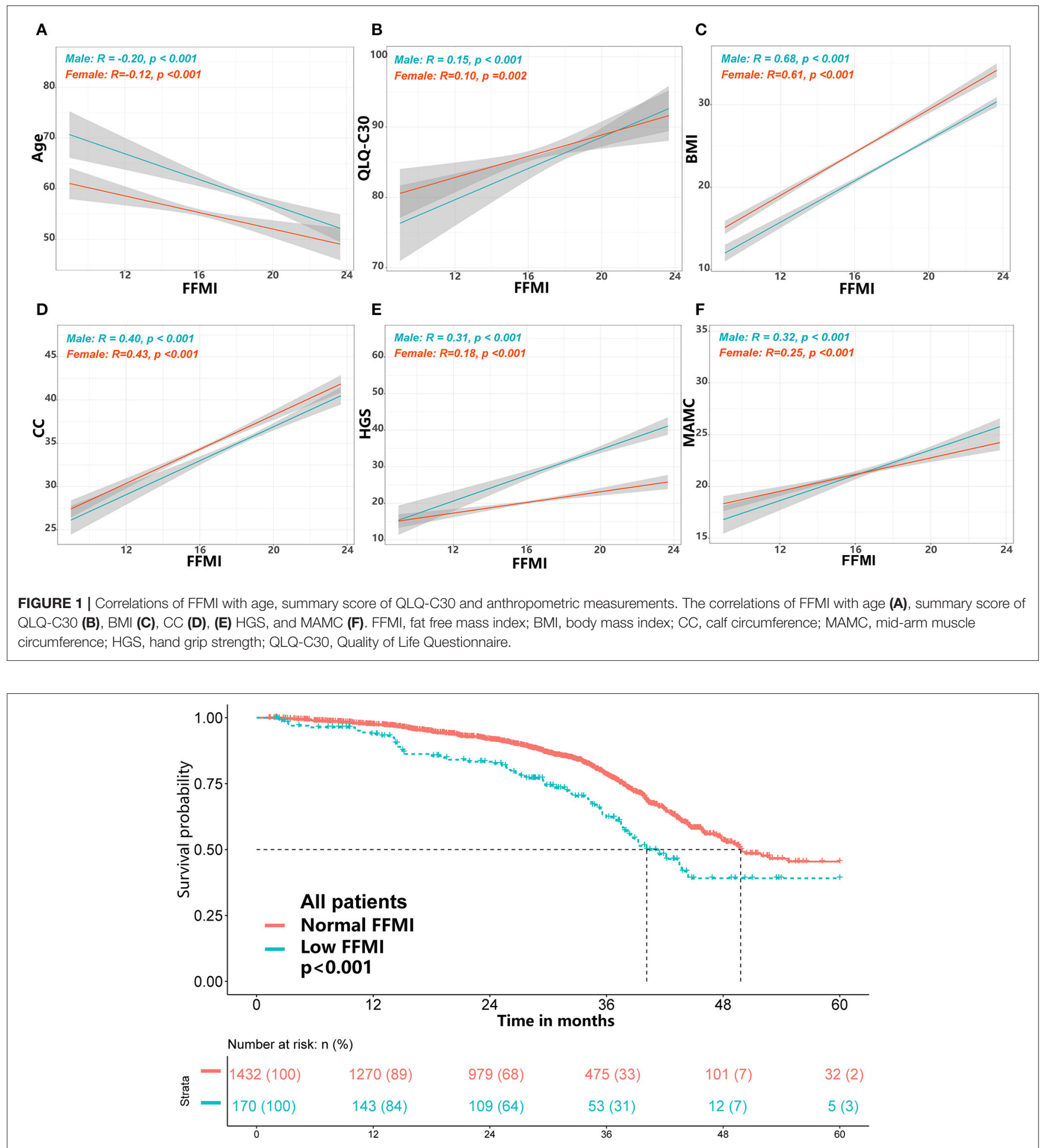

FIGURE 2 | Results of the Kaplan-Meier survival analysis stratified by FFMl in cancer patients with normal/high BMI. 
TABLE 2 | Univariate and multivariate Cox regression analysis of OS in cancer patients with normal/high BMI.

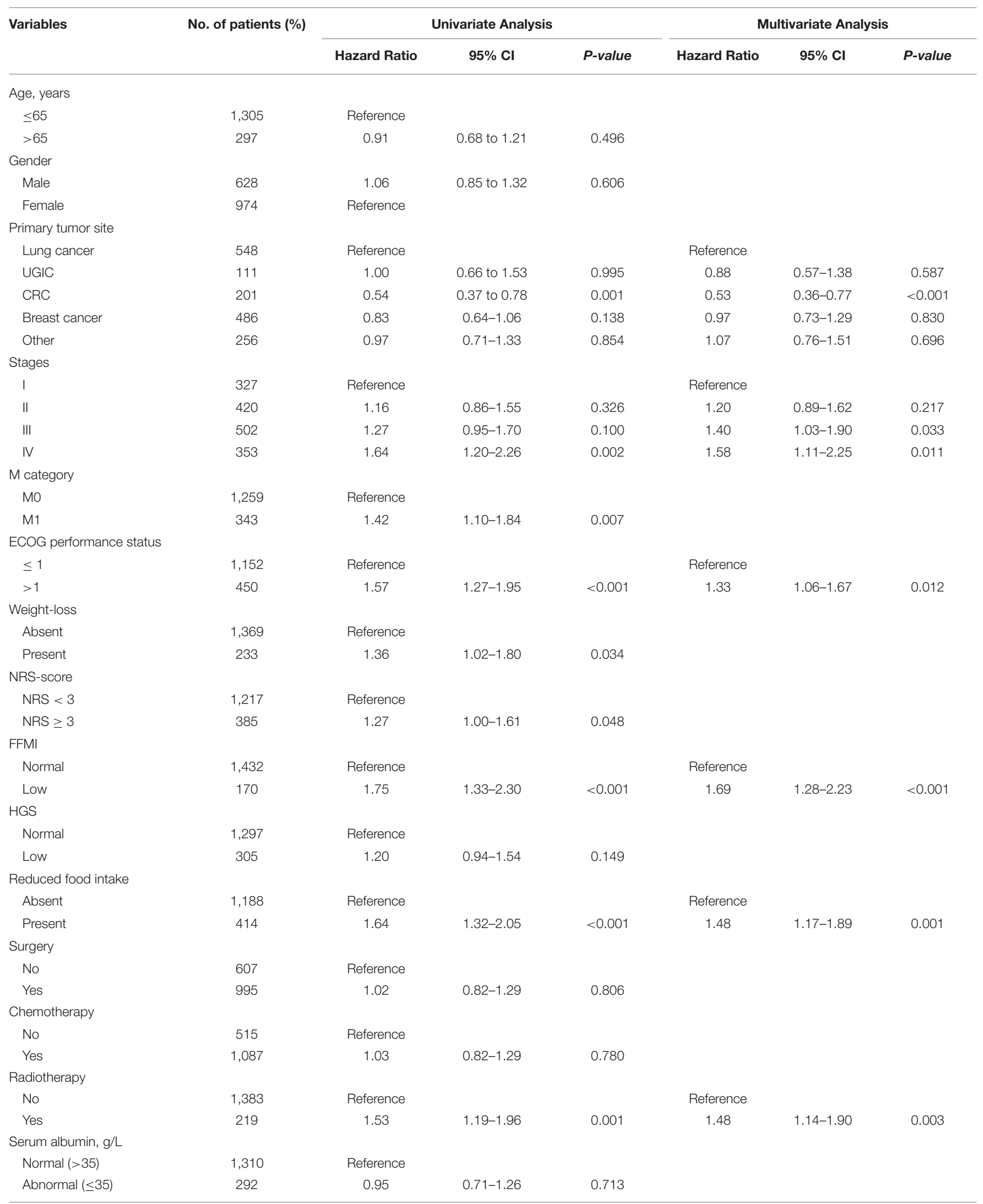


TABLE 2 | Continued

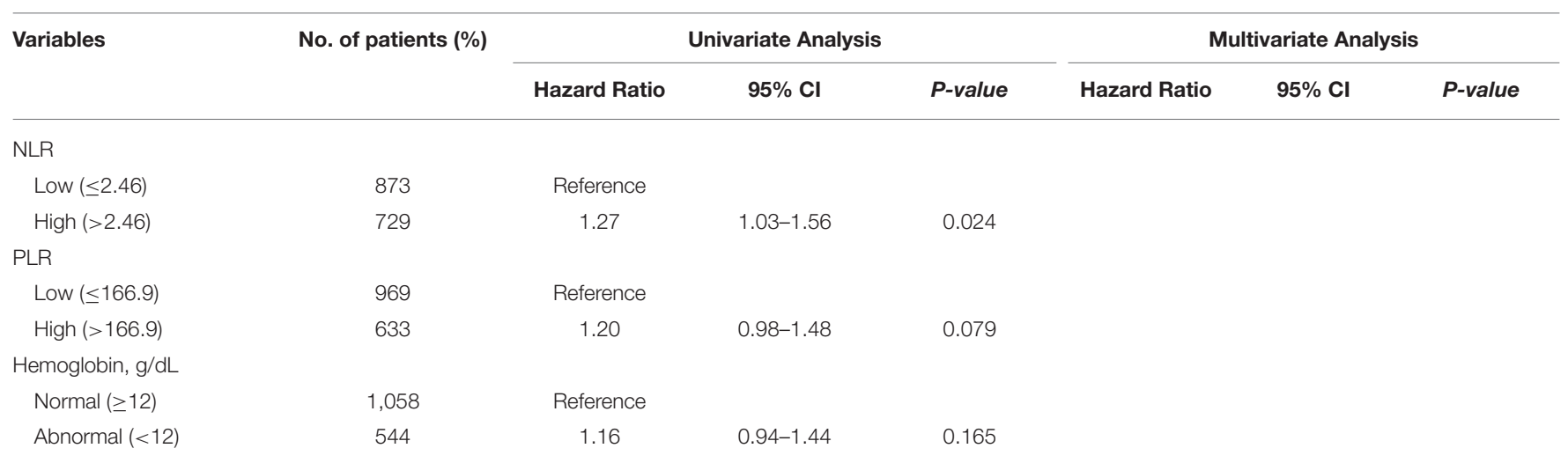

UGIC, upper gastrointestinal cancer; CRC, colorectal cancer; M, metastasis; ECOG, Eastern Cooperative Oncology Group; M, metastasis; FFMI, fat free mass index; NLR, neutrophil-to-lymphocyte ratio; PLR, platelet-to-lymphocyte ratio.

ECOG performance and QLQ-C30 summary score, high NRSscore, metastatic tumors, low hemoglobin, high NLR and PLR, receiving radiotherapy, and upper gastrointestinal cancer (UGIC). In addition, weight loss, reduced food intake, low HGS, low MAMC, low CC and low TSF were more frequently seen in patients with low FFMI (Table 1).

\section{Association of the FFMI With Overall Survival}

Of the 1,602 eligible patients, $10.6 \%$ had a low FFMI. Low FFMI was associated with shorter OS than normal FFMI (Figure 2). Multivariate analysis identified low FFMI as an unfavorable prognostic factor for OS (HR: 1.69; 95\% CI: 1.28, 2.23; $P<$ 0.001) after adjustment for TNM stage, tumor type, ECOG performance status, NLR, weight loss, reduced food intake and radiotherapy (Table 2). Low FFMI was also confirmed as an independent prognostic factor using the sensitivity analysis by excluding patients who died within 6 months or the propensity score-matching analysis (Supplementary Figure 3 and Supplementary Table 1). In addition, low FFMI was significantly associated with poorer OS in patients with lung cancer and breast cancer, and tended to be associated with shorter OS in patients with UGIC (Supplementary Figure 4). After multivariate adjustment, low FFMI was an independent prognostic factor for OS in patients with lung cancer, breast cancer and UGIC, but not for these patients with colorectal cancer (Figure 3). Furthermore, the Kaplan-Meier analysis showed that low FFMI was significantly associated with worse OS in patients received surgery and chemotherapy, and was likely to be associated with shorter OS in patients received radiotherapy (Supplementary Figure 5). Multivariate analysis confirmed low FFMI as an independent worse prognostic factor for OS in patients received surgery and chemotherapy (Figure 3).

\section{Stratified Analyses by Potential Modifiers}

To further elucidate the potential effect of FFMI on prognosis, stratified analyses were performed in several subgroups. The association of low FFMI with worse survival was significantly modified by weight loss $(P$ for interaction $=0.012)$, but not by other potential modifiers including age $(P$ for interaction $=$ $0.611)$, gender $(P$ for interaction $=0.643)$, primary tumor site $(P$ for interaction $=0.962)$, TNM stage $(P$ for interaction $=0.066)$, ECOG performance status $(P$ for interaction $=0.718)$, NLR $(P$ for interaction $=0.149)$, reduced food intake $(P$ for interaction $=0.704)$, surgery $(P$ for interaction $=0.192)$, chemotherapy ( $P$ for interaction $=0.567)$ and radiotherapy $(P$ for interaction $=0.729$ ). When combined with weight loss, low FFMI-weight loss was associated with the worst OS. Intriguingly, weight loss may not confer the poorer prognosis when FFMI was normal (Figure 4). Multivariate analysis, including age, gender, TNM stage, tumor type, ECOG, NLR, weight loss, reduced food intake and radiotherapy, identified the combined presence of low FFMI with weight loss (HR: 3.53; 95\% CI:2.04, 6.11; $p<0.001$ ) and low FFMI without weight loss (HR: 1.45; 95\% CI:1.05, $1.99 ; p=0.022$ ) as significant worse prognostic factors for OS (Supplementary Table 2).

\section{DISCUSSION}

This study was a large-scale study to examine the relationship between low FFMI and mortality in cancer patients with normal or high BMI. Although the GLIM criteria recommended cut-off values for a low FFMI (FFMI $<15 \mathrm{~kg} / \mathrm{m}^{2}$ in women and $<17$ $\mathrm{kg} / \mathrm{m}^{2}$ in men) based on Swiss reference material (9), it might be reasonable that Asians are more likely to have lower FFMI values than Westerns. Moreover, cut-off values of low FFMI need to be linked to the fact that female usually have a lower FFMI and higher FMI than male. Therefore, the current study calculated sex-specific cut-off points of FFMI as $14.5 \mathrm{~kg} / \mathrm{m}^{2}$ for females and $16.3 \mathrm{~kg} / \mathrm{m}^{2}$ for males. Using the cut-off points, low FFMI was found in $10.6 \%$ of cancer patients with normal/high BMI, which is slightly higher than the percentage reported in previous study. Willemsen et al. (21) found that the incidence of low FFMI was $8.7 \%$ in locally advanced head and neck squamous cell carcinoma patients (LAHNSCC) with normal BMI $(\geq 21$ $\mathrm{kg} / \mathrm{m}^{2}$ ). In addition, the results of the present study showed that low FFMI was an independent prognostic factor in the settings of lung cancer, breast cancer and UGIC. In line with 


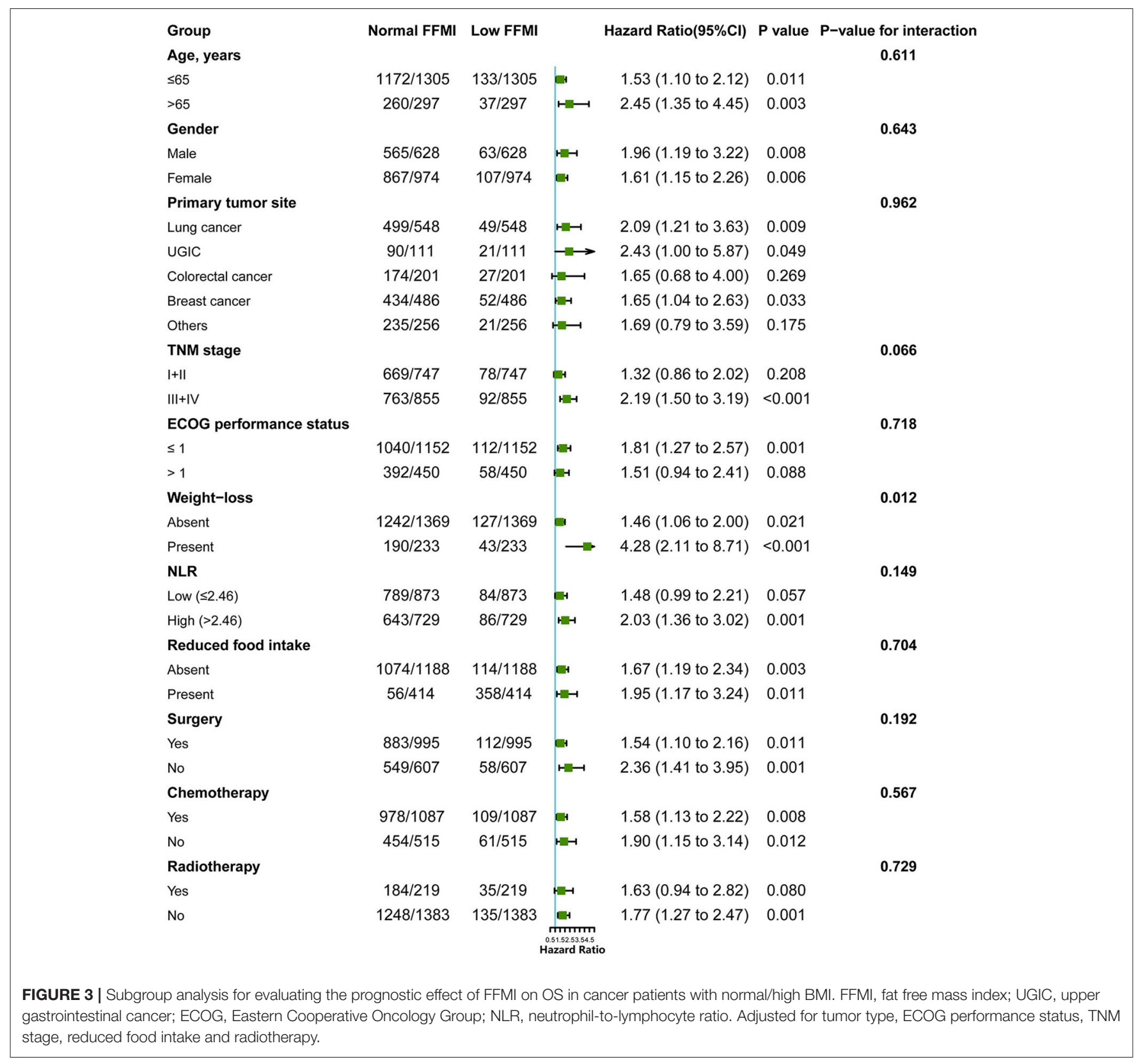

our result, Burtin et al. (22) found low FFMI was associated with worse prognosis in non-small cell lung cancer patients with good performance status (WHO PS 0 or 1). Song et al. (23) demonstrated higher skeletal muscle volume was associated with more favorable prognosis than those with lower muscle volume in patients with breast cancer. Furthermore, we found low FFMI independently associated with worse prognosis in cancer patients received surgery and chemotherapy, but tended to be a worse prognostic factor for patients treated with radiotherapy. One potential reason might be the limited number of patients with low FFMI in radiotherapy group. A recent study found low FFMI was an unfavorable prognostic factor for OS in LAHNSCC patients undergoing chemoradiation or bioradiation treatment (21).
Muscle mass reduction is driven by a variable combination of decreased calorie/protein intake, metabolic changes and inflammation (1). This fact is also partly reflected in the current study that a low FFMI was more frequently seen in patients at risk of malnutrition (NRS score $\geq 3$ ) and in those with high levels of inflammatory biomarkers (high NLR and PLR). Moreover, muscle loss could be linked to a poor prognosis not only pathophysiologically but also indirectly by reducing daily activities (24). The current study found patients with low FFMI usually had poorer ECOG performance and summary score of QLQ-C30. Furthermore, a recent study reported a significant association between low FFMI and accelerated hospitalization in colorectal cancer patients (25). One explanation of this 


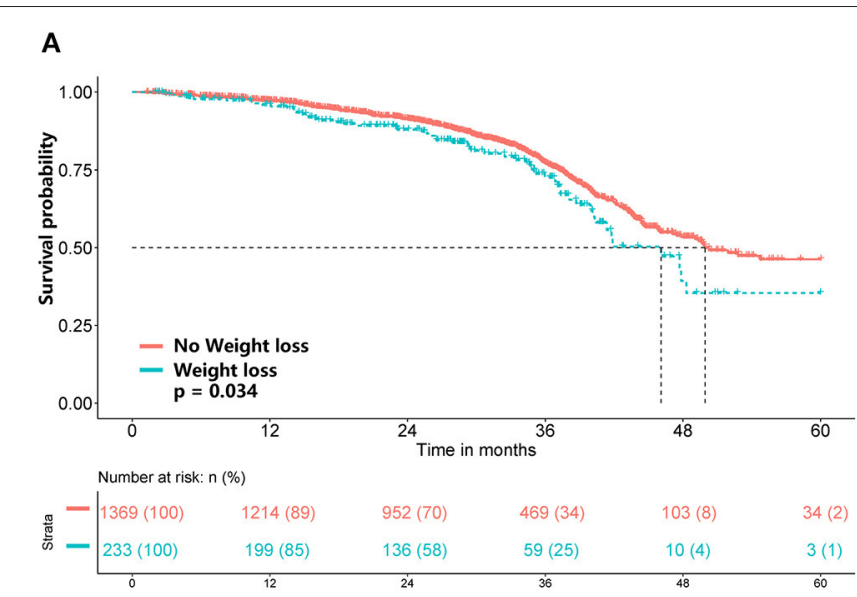

B

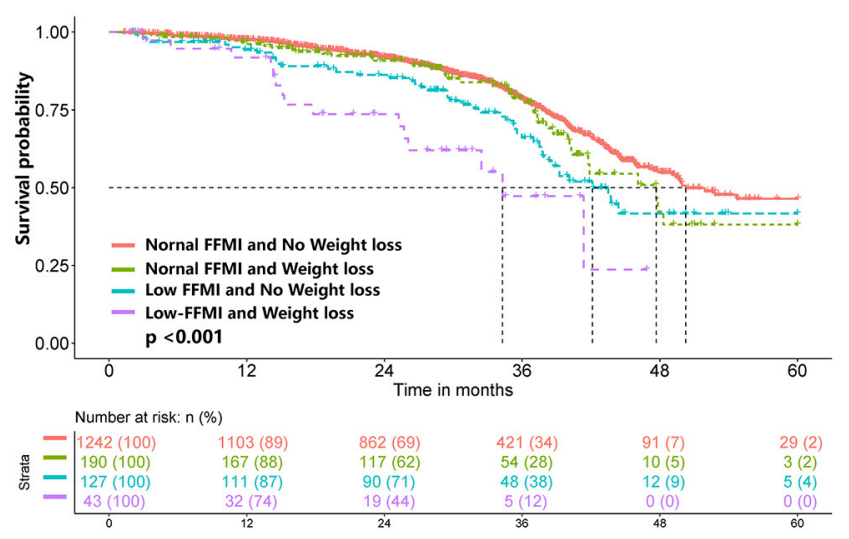

FIGURE 4 | Kaplan-Meier survival analysis of weight loss (A) and FFMl combined with weight loss (B). FFMI, fat free mass index.

phenomenon is that patients with low FFMI have decreased metabolically active body cell mass, which is needed to withstand operative stress and complication development.

The present study found that weight loss combined with low FFMI was associated with worse OS in cancer patients with normal/high BMI. However, there was no significant difference in survival between patients with and without weight loss in the subgroup with normal FFMI, indicating that the FFMI might well represent the association of weight loss and survival. Similarly, a prospective cohort study found that critical weight loss patients with low FFMI had a higher mortality risk, but critical weight loss and normal FFMI patients did not (26). These findings suggest that weight loss only becomes relevant when protein reserves are depleted. In addition, advanced stages of cancer are often associated with altered energy expenditure and lower dietary intake, which can affect body composition. In the present study, the prognostic value of a low FFMI tended to be more pronounced in patients with advanced TNM stages (III and IV). A recent systematic review also reported that low muscle mass was associated with poorer OS in patients with incurable cancer (27). These results emphasize the significance of assessing the FFMI in these patients with advanced TNM stages. In addition, evidence suggests that muscle loss, when combined with increased systemic inflammatory response, worsens the clinical outcomes of cancer patients (28-30). In the present study, we found that the association between low FFMI and poorer prognosis tended to be modified by NLR; low FFMI exhibited more pronounced prognostic significance in patients with high NLR.

Some limitations are associated with the current study. First, FFMI data were retrospectively collected, and were not available for some patients, therefore, the results of this study might be subject to selection bias. However, the relatively large sample size might partially compensate for this limitation. Second, computed tomography (CT) is considered the gold standard in measuring body composition, but was not available in the present cohort. However, BIA measurement is a feasible, non-invasive measure with low cost, and could become widely available in clinical research settings. Finally, due to the limited number of low FFMI cases in overweight and obese BMI subsetting, subgroup analysis could not be conducted in these patients.

In conclusion, this study found low FFMI was associated with worse prognosis in cancer patients with normal/high BMI. The influence of low FFMI on worse prognosis is likely to be more pronounced in patients with weight loss. This study highlights the usefulness of FFMI for prognostic estimation in cancer patients with normal/high BMI.

\section{DATA AVAILABILITY STATEMENT}

The raw data supporting the conclusions of this article will be made available by the authors, without undue reservation.

\section{ETHICS STATEMENT}

The studies involving human participants were reviewed and approved by This study was approved by the Medical Ethics Committee of First Affiliated Hospital of Sun yat-sen University, and registered in the Chinese Clinical Trial Registry (NO ChiCTR1800020329, http://www.chictr.org.cn/). Written informed consent for participation was not required for this study in accordance with the national legislation and the institutional requirements.

\section{AUTHOR CONTRIBUTIONS}

H-PS: conceptualization and methodology. XZ: data curation and writing the original draft preparation. QZ: visualization, investigation, and data curation. L-jF: methodology and investigation. K-PZ: software. MT: validation and visualization. $\mathrm{M}-\mathrm{mS}, \mathrm{G}-\mathrm{tR}$, and X-wZ: writing-reviewing and editing. WL, F-xZ, and M-HC: supervision and investigation. All authors contributed to the article and approved the submitted version. 


\section{FUNDING}

This work was supported by the National Key Research and Development Program (2017YFC1309200). All sources of funding received for the research have been submitted.

\section{REFERENCES}

1. Baracos VE, Martin L, Korc M, Guttridge DC, Fearon KCH. Cancer-associated cachexia. Nat Rev Dis Primers. (2018) 4:17105. doi: 10.1038/nrdp.2017.105

2. Cruz-Jentoft AJ, Bahat G, Bauer J, Boirie Y, Bruyère O, Cederholm $\mathrm{T}$, et al. Sarcopenia: revised European consensus on definition and diagnosis. Age Ageing. (2019) 48:16-31. doi: 10.1093/ageing/a fy169

3. Prado CM, Baracos VE, McCargar LJ, Reiman T, Mourtzakis M, Tonkin K, et al. Sarcopenia as a determinant of chemotherapy toxicity and time to tumor progression in metastatic breast cancer patients receiving capecitabine treatment. Clin Cancer Res. (2009) 15:2920-6. doi: 10.1158/1078-0432.CCR-08-2242

4. Prado CM, Lieffers JR, McCargar LJ, Reiman T, Sawyer MB, Martin L, et al. Prevalence and clinical implications of sarcopenic obesity in patients with solid tumours of the respiratory and gastrointestinal tracts: a population-based study. Lancet Oncol. (2008) 9:629-35. doi: 10.1016/S1470-2045(08)70153-0

5. Bozzetti F. Forcing the vicious circle: sarcopenia increases toxicity, decreases response to chemotherapy and worsens with chemotherapy. Ann Oncol. (2017) 28:2107-18. doi: 10.1093/annonc/mdx271

6. Strulov Shachar S, Williams GR. The obesity paradox in cancer-moving beyond BMI. Cancer Epidemiol Biomarkers Prev. (2017) 26:13-16. doi: 10.1158/1055-9965.EPI-16-0439

7. Schutz Y, Kyle UU, Pichard C. Fat-free mass index and fat mass index percentiles in Caucasians aged 18-98 y. Int J Obes Relat Metab Disord. (2002) 26:953-60. doi: 10.1038/sj.ijo.0802037

8. Kyle UG, Schutz Y, Dupertuis YM, Pichard C. Body composition interpretation. Contributions of the fat-free mass index and the body fat mass index. Nutrition. (2003) 19:597-604. doi: 10.1016/S0899-9007(03)0 0061-3

9. Cederholm T, Jensen GL, Correia MITD, Gonzalez MC, Fukushima R, Higashiguchi T, et al. GLIM criteria for the diagnosis of malnutrition - A consensus report from the global clinical nutrition community. Clin Nutr. (2019) 38:1-9. doi: 10.1016/j.clnu.2019.02.033

10. Martin L, Senesse P, Gioulbasanis I, Antoun S, Bozzetti F, Deans C, et al. Diagnostic criteria for the classification of cancer-associated weight loss. J Clin Oncol. (2015) 33:90-9. doi: 10.1200/JCO.2014.56.1894

11. Shepshelovich D, Xu W, Lu L, Fares A, Yang P, Christiani D, et al. Body mass index (BMI), BMI change, and overall survival in patients with SCLC and NSCLC: a pooled analysis of the international lung cancer consortium. J Thorac Oncol. (2019) 14:1594-607. doi: 10.1016/j.jtho.2019. 05.031

12. Caan BJ, Cespedes Feliciano EM, Kroenke CH. The importance of body composition in explaining the overweight paradox in cancerCounterpoint. Cancer Res. (2018) 78:1906-12. doi: 10.1158/0008-5472.CAN17-3287

13. Franssen FM, Rutten EP, Groenen MT, Vanfleteren LE, Wouters EF, Spruit MA. New reference values for body composition by bioelectrical impedance analysis in the general population: results from the UK Biobank. J Am Med Dir Assoc. (2014) 15:448.e1-6. doi: 10.1016/j.jamda.2014.03.012

14. Chen C, Lu FC, Department of Disease Control Ministry of Health, PR China. The guidelines for prevention and control of overweight and obesity in Chinese adults. Biomed Environ Sci. (2004). 17(Suppl.):1-36.

15. Ma C, Bandukwala S, Burman D, Bryson J, Seccareccia D, Banerjee $\mathrm{S}$, et al. Interconversion of three measures of performance status: an

\section{SUPPLEMENTARY MATERIAL}

The Supplementary Material for this article can be found online at: https://www.frontiersin.org/articles/10.3389/fnut.2021. 714051/full\#supplementary-material

empirical analysis. Eur J Cancer. (2010) 46:3175-83. doi: 10.1016/j.ejca.2010. 06.126

16. Aaronson NK, Ahmedzai S, Bergman B, Bullinger M, Cull A, Duez NJ, et al. The european organization for research and treatment of cancer QLQ-C30: a quality-of-life instrument for use in international clinical trials in oncology. J Natl Cancer Inst.(1993) 85:365-376. doi: 10.1093/jnci/85. 5.365

17. Nordin K, Steel J, Hoffman K, Glimelius B. Alternative methods of interpreting quality of life data in advanced gastrointestinal cancer patients. Br J Cancer. (2001) 85:1265-72. doi: 10.1054/bjoc. 2001.2046

18. Kyle UG, Bosaeus I, De Lorenzo AD, Deurenberg P, Elia M, Manuel Gómez J, et al. Bioelectrical impedance analysis-part II: utilization in clinical practice. Clin Nutr. (2004) 23:1430-53. doi: 10.1016/j.clnu.200 4.09.012

19. Lausen B, Schumacher M. Maximally selected rank statistics. Biometrics. (1992) 48:73-85. doi: 10.2307/2532740

20. Cepeda MS, Boston R, Farrar JT, Strom BL. Comparison of logistic regression versus propensity score when the number of events is low and there are multiple confounders. Am J Epidemiol. (2003) 158:280-7. doi: 10.1093/aje/kwg115

21. Willemsen ACH, Hoeben A, Lalisang RI, Van Helvoort A, Wesseling FWR, Hoebers F, et al. Disease-induced and treatment-induced alterations in body composition in locally advanced head and neck squamous cell carcinoma. J Cachexia Sarcopenia Muscle. (2020) 11:145-59. doi: 10.1002/jcsm. 12487

22. Burtin C, Bezuidenhout J, Sanders KJC, Dingemans AC, Schols AMWJ, Peeters STH, et al. Handgrip weakness, low fat-free mass, and overall survival in non-small cell lung cancer treated with curative-intent radiotherapy. J Cachexia Sarcopenia Muscle. (2020) 11:424-31. doi: 10.1002/jcsm. 12526

23. Song EJ, Lee CW, Jung SY, Kim BN, Lee KS, Lee $\mathrm{S}$, et al. Prognostic impact of skeletal muscle volume derived from crosssectional computed tomography images in breast cancer. Breast Cancer Res Treat. (2018) 172:425-36. doi: 10.1007/s10549-0184915-7

24. Nipp RD, Fuchs G, El-Jawahri A, Mario J, Troschel FM, Greer JA, et al Sarcopenia is associated with quality of life and depression in patients with advanced cancer. Oncologist. (2018) 23:97-104. doi: 10.1634/theoncologist. 2017-0255

25. Tsaousi G, Kokkota S, Papakostas P, Stavrou G, Doumaki E, Kotzampassi K. Body composition analysis for discrimination of prolonged hospital stay in colorectal cancer surgery patients. Eur J Cancer Care (Engl). (2017) 26:e12491. doi: 10.1111/ecc. 12491

26. de van der Schueren MAE, de Smoker M, Leistra E, Kruizenga HM. The association of weight loss with one-year mortality in hospital patients, stratified by BMI and FFMI subgroups. Clin Nutr. (2018) 37:1518-25. doi: 10.1016/j.clnu.2017.08.024

27. Wiegert EVM, de Oliveira LC, Calixto-Lima L, Borges NA, Rodrigues J, da Mota E Silva Lopes MS, et al. Association between low muscle mass and survival in incurable cancer patients: a systematic review. Nutrition. (2020) 72:110695. doi: 10.1016/j.nut.2019. 110695

28. Borges TC, Gomes TL, Pichard C, Laviano A, Pimentel GD. High neutrophil to lymphocytes ratio is associated with sarcopenia risk in hospitalized cancer patients. Clin Nutr. (2020) 40: 202-6. doi: 10.1016/j.clnu.2020. 05.005 
29. Malietzis G, Johns N, Al-Hassi HO, Knight SC, Kennedy RH, Fearon $\mathrm{KC}$, et al. Low muscularity and myosteatosis is related to the host systemic inflammatory response in patients undergoing surgery for colorectal cancer. Ann Surg. (2016) 263:320-5. doi: 10.1097/SLA.00000000000 01113

30. Bilen MA, Martini DJ, Liu Y, Shabto JM, Brown JT, Williams M, et al. Combined effect of sarcopenia and systemic inflammation on survival in patients with advanced stage cancer treated with immunotherapy. Oncologist. (2020) 25:e528-35. doi: 10.1634/theoncologist.2019-0751

Conflict of Interest: The authors declare that the research was conducted in the absence of any commercial or financial relationships that could be construed as a potential conflict of interest.
Publisher's Note: All claims expressed in this article are solely those of the authors and do not necessarily represent those of their affiliated organizations, or those of the publisher, the editors and the reviewers. Any product that may be evaluated in this article, or claim that may be made by its manufacturer, is not guaranteed or endorsed by the publisher.

Copyright (c) 2021 Zhang, Zhang, Feng, Zhang, Tang, Song, Ruan, Zhang, Li, Zhou, Cong and Shi. This is an open-access article distributed under the terms of the Creative Commons Attribution License (CC BY). The use, distribution or reproduction in other forums is permitted, provided the original author(s) and the copyright owner(s) are credited and that the original publication in this journal is cited, in accordance with accepted academic practice. No use, distribution or reproduction is permitted which does not comply with these terms. 